\title{
Carbon monoxide protects Neural Stem Cells against iron overload by modulating the crosstalk between Nrf2 and NF-kB Signaling
}

zhengxing Xie ( $\sim$ hayvip@163.com )

The Affiliated Wuxi No.2 People's Hospital of Nanjing Medical University https://orcid.org/0000-00020589-8580

\section{Aiying $\mathrm{Hu}$}

The Affiliated Hospital of Jiangsu University

\section{qing Wang}

The Affiliated Wuxi No.2 People's Hospital of Nanjing University

\section{Zengli Miao}

The Affiliated Wuxi No.2 People's Hospital of Nanjing Medical University

\section{Research}

Keywords: Neural stem cell, apoptosis, stroke, carbon monoxide

Posted Date: February 25th, 2021

DOI: https://doi.org/10.21203/rs.3.rs-216736/v1

License: (a) (1) This work is licensed under a Creative Commons Attribution 4.0 International License. Read Full License 


\section{Abstract}

Background:It is well $\mathrm{CO}$ is an important gas transmitter. Although accumulating evidences have demonstrated pro-survival effects of $\mathrm{CO}$ against various insults, the precise mechanism explaining how neural stem cell is protected by $\mathrm{CO}$ also remains largely unknown.

Methods: After NSCs were challenged by iron overload with or without CO pretreatment, apoptosis of NSCs was tested by Flow cytometry and the level of antioxidant protein was also detected. ROS of NSCs was demonstrated via the fluorescent probe. We applied siRNA to demonstrated role of the protective effect of CO. In invivo experiment, NSCs with or without CO pretreatment were implanted into intracranial hemorrhagic mouse and the survival of NSCs were detected. The significance of the difference among mean values was determined by the Student-Newman-Keuls test or two-way analysis of variance followed by the Bonferroni post hoc test.

Results: CO effect on pro-survival was comparable to that obtained with pharmacological inhibitors of ROS. Its effect on pro-survival was accompanied by the inhibition of ROS and subsequent inhibition of NF-KB. Such effect is believed to be mediated through Nrf2, in that siRNA-mediated inhibition of Nrf2 revealed that Nrf2 is required for the pro-survival effect of $\mathrm{CO}$. $\mathrm{CO}$ also activated NQO-1, a downstream target of Nrf2, which was inhibited by down-regulating Nrf2. CO-mediated precondition results in Nrf2 stimulation and NF-KB inhibition, suggesting that these two pathways act in an inverse manner to maintain redox homeostasis. The pro-survival effect of $\mathrm{CO}$ was also further endorsed by the evidences in mouse hemorrhagic stroke models.

Conclusions: Results, by showing that $\mathrm{CO}$ attenuates iron overload induced apoptosis by suppressing the NF-kB signaling via Nrf2-mediated anti-oxidant effect, contribute to the understanding of essential cellular pathways. Our findings reveal $\mathrm{CO}$ preconditioning as a promising treatment strategy to improve efficacy of NSCs transplantation after HS.

\section{Introduction}

Neural stem cell (NSC) is considered to be a highly promising candidate for the therapy of hemorrhagic stroke (HS) because of its self-renew and multi-lineage differentiation characteristics. However, massive loss of donor cells post-engraftment becomes a major impediment that lessens the effectiveness of stem cells therapy. Transplanted cells in the deleterious microenvironment are subjected to various insults [1,2 ]. In some studies, only $1-3 \%$ grafted cells survived after grafting [3,4 ]. Oxidative stress mediated damage in stem cells has gained more attention as one of the vital mechanisms implicated in loss of implanted cells $[5,6]$. Growing evidences indicate that there exists an oxidative component in HS-induced brain injury [7]. Indeed, accumulation of reactive oxygen species (ROS) generated by iron overload after HS, leads to ruining the cellular redox-balance. This event becomes a leading cause of brain damage and cell death after HS. Therefore, targeting iron overload-induced oxidative stress is a promising strategy to improve the clinical efficacy of NSCs therapy after HS. 
Endogenous $\mathrm{CO}$ is a major product in heme degradation catalyzed by heme oxygenase 1 (HO-1) and it is considered to play a pivotal role in the protection effect of HO-1. CO has been recognized as an important gas-transmitter in the heart, lung, liver, and the central nervous system (CNS) [8, 9]. In the CNS, CO regulates various signaling pathways in neurons, astrocytes, and stem cells, resulting in neuroprotective and anti-inflammatory effects after acute or chronic CNS injuries [10,11, 12]. The development of COreleasing molecules (CORMs) provides a safe and specific solution for $\mathrm{CO}$ deliver [13]. Previously, we showed CO decreased expression of cleaved caspase 3 via inhibiting NF-KB activation, which accounted for the protection against iron overload-induced damage in NSCs [14]. Nevertheless, the molecular mechanisms explaining how the NF-KB pathway is modulated by $\mathrm{CO}$ also remain largely unknown.

Here, we aimed to elucidate whether and how the cytoprotection by $\mathrm{CO}$ preconditioning is the result of a cross-talk between Nrf2 and NF-kB signaling pathways that leads to anti-oxidative and pro-survival effects. Furthermore, we provided in vivo evidences to support the pro-survival effects of CO on NSCs.

\section{Materials And Methods}

CORM-2 (288144) and N-acetyl-cysteine (NAC) (A7250-10G) were purchased from Sigma-Aldrich (St. Louis, MO, USA).CORM-2 was dissolved in dimethyl sulfoxide, and then diluted in culture media to achieve the required concentrations. Inactivated CORM-2 (iCORM-2) was prepared by dissolving CORM-2 under the same conditions for 3 days at room temperature to liberate all $\mathrm{CO}$ from the molecule [14]. NSC C17.2, a stable, fully characterized, mouse stem cell line [15], was kindly provided by Prof. Jin WL (Institute of Nano Biomedicine and Engineering, Shanghai Jiao Tong University, Shanghai, China). Ferrous chloride $\left(\mathrm{FeCl}_{2}\right)$ was purchased from Sinopharm Chemical Reagent (Shanghai, China). Annexin V-FITC Apoptosis Detection Kit (C1062), Diamidino-2-phenylindole dihydrochloride (DAPI) (C10005) and Reactive Oxygen Species Assay Kit (S0033) were from Beyotime Biotechnology (Shanghai, China). The primary antibodies Nrf2 (C-20) (SC-722) and NF-kB P65 (SC-372) were from Santa Cruz Biotechnology (Santa Cruz, CA, USA). Anti-NQ01 antibody (ab2346) was from Abcam (Cambridge, UK). B-Actin (4970),Tublin(2148) and H3 (12167), were from Cell Signaling Technology (USA).

\section{In vitro experiment}

\section{Cell Culture And Treatment}

C17.2 cells were cultured in Dulbecco's modified Eagle's medium (Invitrogen, USA) containing10 \% (v/v) fetal bovine serum (Gibco, Carlsbad, CA, USA), 5 \% (v/v) horse serum (Gibco), and $2 \mathrm{mM}$ L-glutamine (Gibco) at $37^{\circ} \mathrm{C}$ in a humidified incubator supplemented with $5 \%(\mathrm{v} / \mathrm{v}) \mathrm{CO} 2 . \mathrm{C} 17.2$ cells were pretreated with or without $50 \mu \mathrm{M}$ CORM-2 /iCORM-2 for $6 \mathrm{~h}$ prior to stimulation with $500 \mu \mathrm{M} \mathrm{FeCl}_{2}$ for $24 \mathrm{~h}[14]$.

\section{Western blot analysis}

Western blotting was performed using standard techniques. Briefly, C17.2 cells were grown in 6-well cell culture plates $\left(2 \times 10^{5}\right.$ cells $\left./ \mathrm{mL}\right)$. After treatment, Protein was loaded on $8 \%(\mathrm{w} / \mathrm{v})$ or $10 \%(\mathrm{w} / \mathrm{v})$ SDS- 
polyacrylamide gel and protein levels determined by Western blotting using Nrf2 (1:1000),NF-KB p65(1:1000),NQO-1(1:1000) and HRP-conjugated secondary antibody (1:5000).H3(1:5000) antibodies, and Tublin(1:5000) antibodies were used as marker proteins for nuclear and cytosolic extracts respectively.

\section{RNA interference by small interfering RNA (siRNA) of Nrf2}

Pre-designed siRNAs against mouse Nrf2 and nontargeting control-pool siRNA were purchased from GenePharma (Shanghai, China). For Nrf2-siRNA transfection, C17.2 cells were grown in 6-well plates ( $2 \times$ $10^{5}$ cells per well) until the confluence of cells reached approximately $50 \%$. The cells were then subjected to transient transfection with Nrf2-negative control siRNA, Nrf2-siRNA using the siRNA transfection reagent Lipofectamine ${ }^{\mathrm{TM}} 2000$, following the manufacturer's protocol. After $48 \mathrm{~h}$, the transfected cells were exposed to CORM-2/ iCORM-2 for $6 \mathrm{~h}$, followed by lysis buffer for western blot analysis. Nontargeting siRNA construct (NC) was used as negative control.

\section{Intracellular reactive oxygen species (ROS) measurement}

Detection of intracellular oxidative states was performed by using the fluorescent probe 2,7'dichlorofluorescein diacetate (DCFH-DA) [16] Briefly, C17.2 cells were grown in 6-well cell culture plates $\left(2 \times 10^{5}\right.$ cells $\left./ \mathrm{mL}\right)$ and pretreated with CORM-2 $(50 \mu \mathrm{M})$ or iCORM- 2 for $6 \mathrm{~h}$ prior to stimulation with $500 \mu \mathrm{M}$ $\mathrm{FeCl}_{2}$ for $24 \mathrm{~h}$. the DCFH-DA solution $(10 \mu \mathrm{M})$ was added to the suspension of the cells. After incubation with DCFH-DA for for $20 \mathrm{~min}$ at $37^{\circ} \mathrm{C}$, the fluorescence of DCF was quantified using fluorescence microscopy at excitation and emission wavelengths of 488 and $525 \mathrm{~nm}$, respectively. ROS production was expressed as a percentage of the control. The fold-increase of ROS generation was compared with the control cells, which were arbitrarily considered as 1 -fold.

\section{Flow cytometry}

Apoptosis was determined by using Annexin V-FITC apoptosis kits (Beyotime, China). The assays were performed according to the manufacturer's instructions. Briefly, the cells were pretreated with CORM-2 $(50 \mu \mathrm{M})$ or iCORM-2 for $6 \mathrm{~h}$ prior to stimulation with $500 \mu \mathrm{M} \mathrm{FeCl} 2$ for $24 \mathrm{~h}$. After treatment, single cell suspension was incubated with $5 \mu \mathrm{L}$ of Annexin V-FITC and $10 \mu \mathrm{L}$ of PI in $195 \mu \mathrm{L}$ of binding buffer for 15 min at room temperature in the dark. Then, the rates of apoptosis were analyzed by a flow cytometry (BD Biosciences, San Jose, CA). A minimum of 10,000 events were acquired for each sample.

\section{Immunofluorescent staining}

NSCs cultured on eight-well chamber slides poly-D-lysine-coated glass slips in 24-well dishes were washed with PBS and fixed with 4\% paraformaldehyde in PBS for $15 \mathrm{~min}$. Then, cells were incubated for 1 $\mathrm{h}$ in blocking solution (PBS containing $3 \%$ bovine serum albumin and $0.3 \%$ Triton $\mathrm{X}-100$ ). Cells were then incubated with antibodies against Nrf2 (1:200), NF-KB p65 (1:200) at $4{ }^{\circ} \mathrm{C}$ overnight. After three washes, samples were incubated with donkey anti-rabbit IgG (A21207; Alexa Fluor 594, 1:500; Invitrogen) or goat 
anti-rabbit IgG (A11008; Alexa Fluor 488, 1:500; Invitrogen) at $37^{\circ} \mathrm{C}$ for $2 \mathrm{~h}$. Samples were then washed with PBS and cover slipped with Vectashield mounting medium containing DAPI (Vector Laboratories, Burlington, ON, Canada) nuclear counterstain. Samples were mounted onto slides with anti-fade solution and examined under a confocal laser-scanning microscope.

\section{In vivo experiments}

\section{Animals}

All animal procedures were carried out in accordance with the guideline of the institutional animal care and use committee of The Affiliated Wuxi No.2 Peoples' Hospital of Nanjing Medical University, Wuxi, China. Donate NSCs were harvested from homozygous red fluorescent protein transgenic (RFP Tg) mice (C57BL/6- RFP Tg; Model Animal Research Center of NanJing University, Nanjing, China). Wild-type C57BL/ 6 mice (Slac laboratory animal CO.LTD, Shanghai, China) were for HS models. All animals were housed on a 12: 12 -h light/dark cycle with environmental temperatures at $18-22^{\circ} \mathrm{C}$. Food and water were freely available.

\section{Isolation and culture of fetal neural stem cells}

NSCs were isolated from the subventricular zones of RFP Tg fetal mice at 14 days of gestation, as described previously [17]. In brief, bilateral subventricular zones were dissected and mechanically dissociated. After collection, the cells were resuspended in Neuro-basal-A medium (Invitrogen, Carlsbad, CA, USA) containing B-27 supplement (Invitrogen), L-glutamine (Invitrogen), 20ng/ml mouse fibroblast growth factor-basic (PeproTech, Rocky Hill, NJ, USA), and $10 \mathrm{ng} / \mathrm{ml}$ mouse epidermal growth factor (PeproTech, Rocky Hill, NJ, USA). Cells were grown as suspending neurosphere. The medium was halfchanged every 2 days and cells were passaged weekly. Cells that had been passaged 5 to 10 times were used for the experiments.

\section{HS model and experiment group}

We used an experimental HS procedure, described previously [2]. Briefly, male C57BL/6 mice (8 weeks old, 20 to $25 \mathrm{~g}$ ) were anesthetized with ketamine/xylazine (100 mg/10 mg/kg, Sigma, St. Louis, MO), and positioned in the stereotactic frame.After a midline scalp incision, a hole was drilled in the right side of the skull $(0.0 \mathrm{~mm}$ anterior and $2.5 \mathrm{~mm}$ lateral to the bregma). Blood $(20 \mu \mathrm{l})$ without any anticoagulant was collected from the tail tip for injection. A 30-gauge needle attached to a 50-ml Hamilton micro-syringe was inserted $3.5 \mathrm{~mm}$ ventral from the surface of the skull. Ten microliters of blood was injected over 10 minutes. The remaining $10 \mathrm{ml}$ of blood was injected using the same procedure. After infusion, the needle was left in place for 25 minutes and then slowly removed.

Experimental groups were non-precondition group, transplantation of RFP-NSCs $\left(3 * 10^{5} / 2 \mu l ; n=15\right)$;

iCORM-2-precondition group, transplantation of iCORM-2 preconditioned RFP-NSCs $\left(3^{\star} 10^{5} / 2 \mu \mathrm{l} ; n=15\right)$ and 
CORM-2-precondition group, transplantation of CORM-2 preconditioned RFP-NSCs $\left(3^{*} 10^{5} / 2 \mu \mathrm{l} ; \mathrm{n}=15\right)$. All methods and assessments described below were carried out by individuals blinded to the groups.

\section{CO preconditioning and Intracerebral transplantation}

CORM-2/iCORM-2 dissolved in DMSO was added to the cell culture medium (final concentration: $50 \mu \mathrm{M}$ ) for $6 \mathrm{~h}$ prior to transplantation, followed by drug washout before transplanting. NSCs were transplanted on the 3rd day after HS as described previously [2].

\section{Assessment of survival of donate NSCs}

HS animals were sacrificed on the thirtieth days after transplantation to detect the implanted NSCs. The survival of donate RFP Tg NSCs were assessed on 12 serial coronal sections per brain $(0.25 \mathrm{~mm}$ apart) using unbiased computational stereology.

\section{Statistical analysis}

Data were analyzed by using SPSS version 18 software. The significance of the difference among mean values was determined by the Student-Newman-Keuls test or two-way analysis of variance followed by the Bonferroni post hoc test. $P$ values $<0.05$ were accepted to be statistically significant.

\section{Results}

\section{The ROS role in apoptosis of NSCs challenged by iron overload}

The present investigation was aimed to determine the role of ROS in iron overload -induced apoptosis in neural stem cells. After $24 \mathrm{~h}$ of iron overload, flow cytometry analysis revealed a significant increase of apoptosis of NSCs up to $21.4 \pm 3.0 \%$ VS control cells $1.9 \pm 0.4 \%(\mathrm{p} \otimes 0.05)$. As the results showed in Fig1, cotreatment with ROS inhibitor N-acetylcysteine (NAC $1 \mathrm{mM}$ ) protected NSCs against iron overload-induced cell apoptosis and the apoptosis rate decreased from $21.4 \pm 3.0 \%$ to $7.5 \pm 0.8 \%$ ( $p \bowtie 0.05$ ).

\section{Iron overload induced activation of NF-KB signaling pathway via ROS}

Our previous study demonstrated iron overload induced significant activation of NFKB [14]. To confirm the role of ROS on iron overload-induced NFKB activation, immunofluorescence was performed to detect the nuclear translocation of $\mathrm{NFKB}$ (Fig.1E). We found that NFKB was tethered in cytoplasm in un-stimulated control cells, but in those cells challenged by $\mathrm{FeCL}_{2}$, the nuclear translocation of $\mathrm{NFKB}$ was significant. In NSCs co-treated with ROS inhibitor (NAC 1 mM) the translocation was inhibited. For further confirmation of ROS role, next we performed Western blot and examined the changes in NFKB. We found that iron overload -induced up-regulation of NFKB in nucleus fraction was substantially suppressed by NAC (Fig.1G). These findings strongly demonstrated that ROS signaling cascades are involved in the regulation of iron overload -induced NFKB activation. 


\section{CO inhibits iron overload-induced intracellular ROS accumulation}

ROS are known to play a key role in the damage of neural cells after HS. Our in vitro studies demonstrated that iron overload-induced ROS accumulation in NSCs may mediate the increase of apoptosis of NSCs. As depicted in Fig. 2, NSCs subjected to $\mathrm{FeCL}_{2}(500 \mu \mathrm{M})$ for $24 \mathrm{~h}$ triggered the intracellular ROS production (pष0.001). However, the aberrant accumulation of ROS was reduced by preconditioning with CORM-2 (50 $\mu \mathrm{M})$ prior to iron overload stimulation ( $\mathrm{p} \otimes 0.05$ ). Despite, cells incubated with iCORM-2 showed little effect on the increased ROS levels compared to CORM-2 pretreatment. This finding suggests that resistance to oxidative stress is one of the mechanisms of CO-mediated cyto-protection.

\section{$\mathrm{CO}$ triggers the nuclear translocation of Nrf2 and up-regulates the level of Nrf2 in nucleus}

Nrf2 is an important transcription factor, which mediate antioxidant responses upon stimulation. Here we examined whether $\mathrm{CO}$ suppressed the iron overload-induced ROS accumulation via activation of Nrf2. Nrf2 is normally located in the cytosol, bound and inhibited by Keap1. Following activation, Nrf2 is released from this inhibition, and translocates to the nucleus and activates transcription of downstream antioxidant genes. To verify this, immunofluorescence was performed to detect the nuclear translocation of Nrf2. We found that Nrf2 was tethered in cytoplasm in un-stimulated control cells, but in those cells preconditioned by CORM-2 such translocation is significant (Fig.3A). For further confirmation of $\mathrm{CO}$ antioxidant effects, next we performed Western blot and examined the changes in nuclear and cytosolic fractions of NSCs. Interestingly, Consistent with nuclear translocation of Nrf2 induced by CO, a significantly increase of the level of Nrf2 in nucleus fractions about 2-3 fold was determined by western blot (Fig.3C).

\section{CO protective effects are associated with enhanced antioxidant genes}

We next investigated whether $\mathrm{CO}$ could stimulate Nrf2 mechanism, with its transcriptional activity being related to phase II antioxidant enzyme genes such as NQO-1 which are responsible for scavenging ROS [18]. We hypothesized that protective effect of $\mathrm{CO}$ may be due to induction of antioxidant genes, NQO-1 through the typical Nrf2/ ARE-signaling cascades. To confirm this, first we measured the time dependent changes in proteins levels of NQO-1after $\mathrm{CO}$ preconditioning. As expected, $\mathrm{CO}$ preconditioning significantly increased NQO-1 protein expressions in a time dependent manner (Fig.4A). The significantly increased NQO-1protein after $\mathrm{CO}$ preconditioning started after treatment for $6 \mathrm{~h}$ (Fig.4A). CO preconditioning increased NQO-1 significantly in NSC challenged by $\mathrm{FeCL}_{2}$ (Fig.4B). Next we downregulate Nrf2 to confirm whether $\mathrm{CO}$ could promote antioxidant genes via Nrf2/AREsignaling pathway. Results showed Nrf2-siRNA significantly down-regulated CO-mediated protein expression of Nrf2, NQO1 (Fig.4C).

\section{CO suppresses NFKB activation through up-regulation of Nrf2}

It has been reported that $\mathrm{CO}$ pretreatment can suppress the activation of NFKB. To delineate the role of Nrf2 signaling cascade on the fate of NFKB, we examined nucleus fraction protein level of NFKB in NSCs 
transfected by siRNA Nrf2. We found that NFKB increased significantly and the inhibition of NFKB by CO was abolished in nucleus fraction after Nrf2 was knocked down (Fig.4D). These findings illustrate that CO-mediated suppression of iron overload-induced NFKB activation was regulated by activation of Nrf2.

\section{Nrf2 knockdown diminishes the protective effects of $\mathrm{CO}$ in iron overload stimulated NSCs}

To reveal the importance of Nrf2 activation behind CO-induced protection against iron overload, we developed an Nrf2 knockdown C17.2 cells using siRNA transfection. The apoptosis of NSCs was determined by flow cytometry. $\mathrm{CO}$ decreased the apoptosis of NSCs induced by iron overload from $24.4 \pm 4.4 \%$ to $7.5 \pm 1.0 \%$. However, CO-mediated protection was abolished in the cells transfected by siRNA Nrf2 with an apoptosis even up to $38.6 \pm 3.1 \%$ (Fig.5A). The protective effect was exclusively for CO because pretreatment with iCORM-2 did not cause any additional effect, i.e. the apoptosis of neural stem cells were unmodified. Concomitant with the apoptosis of NSCs, CO-induced up-regulation of Nrf2 and NQO-1 proteins was barely observed in Nrf2 knockdown cells (Fig.4C). This data suggest that induction of NQO-1 by CO requires Nrf2 activation, which seems to be essential behind $\mathrm{CO}$ antioxidant effects against iron overload.

\section{Quantification of survival of the transplanted red fluorescent protein-positive neural stem cells in vivo}

The transplanted RFP-positive cells were counted on 12 serial coronal sections per brain as described [2].CO preconditioning NSCs showed a significant improvement of survival in HS mouses(Fig.6B)while pretreatment with iCORM-2 did not cause any additional effect(Fig.6C)

\section{Discussion}

For the first time we provide in vitro and in vivo evidences to demonstrate that CO protects implanted NSCs against iron overload -induced redox imbalance in HS. Our results show that CO effectively enhances the survival of NSCs after HS which is likely associated with reestablishing the redox balance via modulation of the crosstalk between Nrf2 and NF-KB signaling pathway.

It has been well established that iron overload was responsible for the adverse outcome in stem cell $[19,20]$.In our study, when NSCs were treated with $\mathrm{FeCL}_{2}$ for $24 \mathrm{~h}$, we found a marked cellular toxic effect consistent with a sustained increase of ROS. Under these experimental conditions, nuclear protein level of NF-KB was increased concomitant with significant translocation. The activation of the NF-KB signaling pathway seems to be responsive to excessive ROS generation, thus affecting the redox sensitive NF-KB signaling pathway [21]. Indeed, considerable demonstrations established the link between oxidative stress and NF-KB which was mainly from the inhibition of NF-KB activation by cellular antioxidants [22,23]. Further evidence in our study showed that iron overload-induced NF-KB activation was inhibited by Nacetylcysteine (NAC) (pharmacological inhibitors of ROS), which implies that iron overload -mediated NF$\mathrm{KB}$ activation in NSC was ROS dependent. A growing body of evidence has demonstrated the deleterious role of the activation of NF-KB induced by iron overload [24,25]. Such deleterious role of the activation of NF-KB has also been shown in PC12 cell and hippocampal neuron cell challenged by other oxidant regent 
[26]. The neuroprotective effect of inhibition of NF-KB was found in NSCs in our research and reproduced in other study [27]. Our previous data indicated pretreatment with $\mathrm{CO}$ could inhibit the activation of NF-KB induced by iron overload. Such inhibition was reasoned by suppression of ROS as evidenced by suppressed ROS in NSCs upon the pretreatment with CO and suppressed NF-KB activation upon treatment via inhibition of ROS with NAC. In this regard, $\mathrm{CO}$ protective effects are similar to these of aspirin, which has been shown to attenuate $\mathrm{H}_{2} \mathrm{O}_{2}$-induced apoptosis in Hela cell through NF-KB inactivation [28]. The present findings further emphasized that CO-mediated suppression of NF-kB activation is associated with the inhibition of iron overload-induced ROS accumulation in NSCs.

The Nrf2 signaling pathway is the main pathway responsible for cell defense against oxidative damage and maintaining the cellular redox balance [29]. Disruption of Nrf2-mediated Nrf2/ARE-signaling pathway could exacerbate the oxidative damage[30]. There are several examples that $\mathrm{CO}$ acts in CNS by activation of a preconditioning state via Nrf2 signaling [31,32]. In our study, the activation of Nrf2 was evidenced by the significant upregulation of the protein level of Nrf2 and the nuclear translocation of Nrf2. Elevated antioxidant enzymes levels by $\mathrm{CO}$ through Nrf2/ARE cascades may play a frontline of defense against iron overload-induced ROS. The expressing of NQO-1 in NSC exhibited in a time-dependent manner after $\mathrm{CO}$ preconditioning. The pivot role of NQO-1 induced by Nrf2 in NSC against oxidative stress has been well established [33]. Nrf2 knockdown (siNrf2) studies confirmed this phenomenon by detecting the unchanged NQO-1 level and significant cell apoptosis even in the presence of CO.

Recently, substantial efforts have been put on the issue that when Nrf2 talks who is listening. It is worthwhile to note that suppression of NF-KB accumulation by $\mathrm{CO}$ was accompanied by activation of Nrf2. Evidences have also been accumulating that suggest that activation of Nrf2 antioxidant signaling occurs concomitantly with suppression of NF-KB response. [34,35]. Furthermore, Nrf2 $2^{-/-}$mice has been demonstrated increased NF-KB activation when compared with wild-type after traumatic brain injury[36]. These findings further emphasize the crosstalk between NFKB and Nrf2 transcription factors [37]. The relationship between Nrf2 and NF-KB signaling pathway is now suggested as an important cross-talk mechanism by which Nrf2 can overcome apoptosis and provide protection against various deleterious stresses $[38,39]$. Several lines of evidence suggest that Nrf2 regulates NF-KB activation through maintenance of redox homeostasis. In the presence of $\mathrm{CO}$, Nrf2 protein expression was up-regulated concomitant with increase of nuclear translocation, which leads to the induction of antioxidant enzymes NQ01 with simultaneous inhibition of iron overload-induced ROS production and a subsequent suppression of the NF-KB. Nrf2-mediated expression of NQ01 has been demonstrated efficiently to neutralize reactive oxygen species (ROS) and to cause the reduction of ROS-mediated NF-KB activation [40]. Notably, siRNA-mediated suppression of Nrf2 expression in NSCs also witnessed that iron overloadinduced elevation of NF-KB levels remains higher and more significant cell apoptosis occurs. Since Nrf2regulated genes are responsible for increased cellular oxidative stress and ROS is involved in iron overload -induced activation of NF-kB, we postulate that Nrf2 activation by $\mathrm{CO}$ may, at least in part, downregulate the iron overload-induced NF-KB through the modulation of redox status. In accordance with our findings, Minellia et al have shown that regulation of the crosstalk between Nrf2 and NF-KB by CO is highly neuroprotective by reducing oxidative stress in PC12 and murine microglial BV2 cells [41]. These 
findings underlined the possibility that $\mathrm{CO}$-mediated neuro-protective effect may be achieved by the modulation of the crosstalk between Nrf2 and NF-KB via reestablishing the redox balance in NSC.

On particular interest of our in vitro findings, we conducted in vivo studies to demonstrate the therapeutic potentials of $\mathrm{CO}$ pretreatment in HS mouse model. The pro-survival effect of $\mathrm{CO}$ was further endorsed by in vivo evidences. In our experiment, survival of more CO preconditioned RFP-NSCs ascertains the protective role of $\mathrm{CO}$. Also, day 3 after HS is an important time point for hematoma evacuation operation in clinic, and NSCs could be implanted at the same time of hematoma evacuation. SO, day 3 after HS is an interesting time point for investigation of the survival of donor NSCs and it has potential implication for clinical therapy [42].

\section{Discussion}

For the first time we provide in vitro and in vivo evidences to demonstrate that $\mathrm{CO}$ protects implanted NSCs against iron overload -induced redox imbalance in HS. Our results show that $\mathrm{CO}$ effectively enhances the survival of NSCs after HS which is likely associated with reestablishing the redox balance via modulation of the crosstalk between Nrf2 and NF-KB signaling pathway.

It has been well established that iron overload was responsible for the adverse outcome in stem cell [19, 20].In our study, when NSCs were treated with $\mathrm{FeCL}_{2}$ for $24 \mathrm{~h}$, we found a marked cellular toxic effect consistent with a sustained increase of ROS. Under these experimental conditions, nuclear protein level of NF-KB was increased concomitant with significant translocation. The activation of the NF-KB signaling pathway seems to be responsive to excessive ROS generation, thus affecting the redox sensitive NF-KB signaling pathway [21]. Indeed, considerable demonstrations established the link between oxidative stress and NF-KB which was mainly from the inhibition of NF-KB activation by cellular antioxidants [22, 23]. Further evidence in our study showed that iron overload-induced NF-KB activation was inhibited by Nacetylcysteine (NAC) (pharmacological inhibitors of ROS), which implies that iron overload -mediated NFKB activation in NSC was ROS dependent. A growing body of evidence has demonstrated the deleterious role of the activation of NF-KB induced by iron overload [24, 25]. Such deleterious role of the activation of NF-KB has also been shown in PC12 cell and hippocampal neuron cell challenged by other oxidant regent [26]. The neuroprotective effect of inhibition of NF-KB was found in NSCs in our research and reproduced in other study [27]. Our previous data indicated pretreatment with $\mathrm{CO}$ could inhibit the activation of NF-KB induced by iron overload. Such inhibition was reasoned by suppression of ROS as evidenced by suppressed ROS in NSCs upon the pretreatment with $\mathrm{CO}$ and suppressed NF-KB activation upon treatment via inhibition of ROS with NAC. In this regard, CO protective effects are similar to these of aspirin, which has been shown to attenuate $\mathrm{H}_{2} \mathrm{O}_{2}$-induced apoptosis in Hela cell through NF-KB inactivation [28]. The present findings further emphasized that $\mathrm{CO}$-mediated suppression of NF-KB activation is associated with the inhibition of iron overload-induced ROS accumulation in NSCs.

The Nrf2 signaling pathway is the main pathway responsible for cell defense against oxidative damage and maintaining the cellular redox balance [29]. Disruption of Nrf2-mediated Nrf2/ARE-signaling pathway 
could exacerbate the oxidative damage[30]. There are several examples that $\mathrm{CO}$ acts in CNS by activation of a preconditioning state via Nrf2 signaling [31, 32]. In our study, the activation of Nrf2 was evidenced by the significant upregulation of the protein level of Nrf2 and the nuclear translocation of Nrf2. Elevated antioxidant enzymes levels by $\mathrm{CO}$ through Nrf2/ARE cascades may play a frontline of defense against iron overload-induced ROS. The expressing of NQO-1 in NSC exhibited in a time-dependent manner after $\mathrm{CO}$ preconditioning. The pivot role of NQO-1 induced by Nrf2 in NSC against oxidative stress has been well established [33]. Nrf2 knockdown (siNrf2) studies confirmed this phenomenon by detecting the unchanged NQO-1 level and significant cell apoptosis even in the presence of $\mathrm{CO}$.

Recently, substantial efforts have been put on the issue that when Nrf2 talks who is listening. It is worthwhile to note that suppression of NF-KB accumulation by $\mathrm{CO}$ was accompanied by activation of Nrf2. Evidences have also been accumulating that suggest that activation of Nrf2 antioxidant signaling occurs concomitantly with suppression of NF-KB response. [34, 35]. Furthermore, $\mathrm{Nrf2}{ }^{-/-}$mice has been demonstrated increased NF-KB activation when compared with wild-type after traumatic brain injury[36]. These findings further emphasize the crosstalk between NFKB and Nrf2 transcription factors [37]. The relationship between Nrf2 and NF-KB signaling pathway is now suggested as an important cross-talk mechanism by which Nrf2 can overcome apoptosis and provide protection against various deleterious stresses $[38,39]$. Several lines of evidence suggest that Nrf2 regulates NF-KB activation through maintenance of redox homeostasis. In the presence of $\mathrm{CO}$, Nrf2 protein expression was up-regulated concomitant with increase of nuclear translocation, which leads to the induction of antioxidant enzymes NQ01 with simultaneous inhibition of iron overload-induced ROS production and a subsequent suppression of the NF-KB. Nrf2-mediated expression of NQ01 has been demonstrated efficiently to neutralize reactive oxygen species (ROS) and to cause the reduction of ROS-mediated NF-KB activation [40]. Notably, siRNA-mediated suppression of Nrf2 expression in NSCs also witnessed that iron overloadinduced elevation of NF-KB levels remains higher and more significant cell apoptosis occurs. Since Nrf2regulated genes are responsible for increased cellular oxidative stress and ROS is involved in iron overload -induced activation of NF-kB, we postulate that Nrf2 activation by $\mathrm{CO}$ may, at least in part, downregulate the iron overload-induced NF-kB through the modulation of redox status. In accordance with our findings, Minellia et al have shown that regulation of the crosstalk between Nrf2 and NF-KB by CO is highly neuroprotective by reducing oxidative stress in PC12 and murine microglial BV2 cells [41]. These findings underlined the possibility that $\mathrm{CO}$-mediated neuro-protective effect may be achieved by the modulation of the crosstalk between Nrf2 and NF-KB via reestablishing the redox balance in NSC.

On particular interest of our in vitro findings, we conducted in vivo studies to demonstrate the therapeutic potentials of $\mathrm{CO}$ pretreatment in HS mouse model. The pro-survival effect of $\mathrm{CO}$ was further endorsed by in vivo evidences. In our experiment, survival of more CO preconditioned RFP-NSCs ascertains the protective role of $\mathrm{CO}$. Also, day 3 after HS is an important time point for hematoma evacuation operation in clinic, and NSCs could be implanted at the same time of hematoma evacuation. SO, day 3 after HS is an interesting time point for investigation of the survival of donor NSCs and it has potential implication for clinical therapy [42]. 


\section{Conclusion}

Our findings demonstrated that CO preconditioning reprograms NSCs to tolerate oxidative stress induced by iron overload after HS. The anti-oxidant effects of $\mathrm{CO}$ are mediated through the activation of $\mathrm{Nrf} 2$ and subsequent up-regulation of antioxidant genes NQO-1. Our data directly implies that CO protects NSCs against iron overload by modulating the crosstalk between Nrf2 and NF-KB via reestablishing intracellular redox balance. Through this better understanding of $\mathrm{CO}$-mediated signal transduction and gene regulations involved in redox status, our findings may provide an opportunity to improve the efficacy of NSCs therapy after HS.

\section{Declarations}

Ethics approval and consent to participate: The study was approved by the Ethical committee of The Affiliated Wuxi No.2 Peoples' Hospital of Nanjing Medical University, Wuxi, China

Consent for publication: All authors consent for publication

\section{Availability of data and material: $Y$}

Competing interests: All authors declare no conflicts of interests

Funding: Nanjing Medical University Foundation (Grant No.2017NJMUD114) ,Wuxi Municipal Bureau Foundation (Grant No.N20192022) and Top Talent Support Program for young and middle-aged people of Wuxi Health Committee (Grant No.HB2020036)

Authors' contributions: Designs and funding (Xie Zhengxing); Cell culture and treatment, Western blot analysis(Wang Qing); RNA interference and Flow cytometry(Xie Zhengxing, Hu Aiying); Immunofluorescent staining, Isolation and culture of fetal neural stem cells, HS model and Intracerebral transplantation(Xie Zhengxing, Hu Aiying); Statistical analysis(Xie Zhengxing,Miao Zengli)

Acknowledgements: All authors gratefully acknowledge Prof. Jin WL for kind provision of C17.2 NSCs.

\section{Abbreviations}

CNS central nervous system

CO carbon monoxide

CORMs co-releasing molecules

DCFH-DA 2',7'-dichlorofluorescein diacetate

$\mathrm{FeCl}_{2} \quad$ Ferrous chloride 
hemorrhagic stroke

NAC N-acetylcysteine

NF-kB nuclear factor kappa-B

Nrf2 nuclear factor erythroid 2-related factor 2

NSC neural stem cell

RFP Tg fluorescent protein transgenic

ROS reactive oxygen species

siRNA RNA interference by small interfering RNA

\section{References}

1. Othman FA, Tan SC. Preconditioning Strategies to Enhance Neural Stem Cell-Based Therapy for Ischemic Stroke. Brain Sci. 2020 Nov 23;10(11):893.

2. Wakai T, Narasimhan P, Sakata H, Wang E, Yoshioka H, Kinouchi H, Chan PH. Hypoxic preconditioning enhances neural stem cell transplantation therapy after intracerebral hemorrhage in mice J Cereb Blood Flow Metab. 2015 Oct 27. pii: 0271678X15613798. [Epub ahead of print].

3. Hicks AU, Lappalainen RS, Narkilahti S, Suuronen R, Corbett D, Sivenius J, Hovatta O, Jolkkonen J. Transplantation of human embryonic stem cell-derived neural precursor cells and enriched environment after cortical stroke in rats: cell survival and functional recovery. Eur $\mathrm{J}$ Neurosci. 2009;29(3):562-74.

4. Nakagomi N, Nakagomi T, Kubo S, Nakano-Doi A, Saino O, Takata M, Yoshikawa H, Stern DM, Matsuyama T, Taguchi A. Endothelial cells support survival, proliferation, and neuronal differentiation of transplanted adult ischemia-induced neural stem/progenitor cells after cerebral infarction. Stem Cells. 2009;27(9):2185-95.

5. Yagi H, Tan J, Tuan RS. Polyphenols suppress hydrogen peroxide-induced oxidative stress in human bone-marrow derived mesenchymal stem cells. J Cell Biochem. 2013;114(5):1163-73.

6. Cooke MS, Evans MD, Dizdaroglu M, Lunec J. Oxidative DNA damage: mechanisms, mutation, and disease. FASEB J. 2003;17(10):1195-214.

7. Qureshi Al1, Mendelow AD, Hanley DF. Intracerebral haemorrhage. Lancet. 2009;373(9675):1632-44.

8. Rochette L, Cottin Y, Zeller M, Vergely C. Carbon monoxide: mechanisms of action and potential clinical implications. Pharmacol Ther. 2013;137(2):133-52.

9. Deng J, Lei C, Chen Y, Fang Z, Yang Q, Zhang H, Cai M, Shi L, Dong H, Xiong L. Neuroprotective gases-fantasy or reality for clinical use? Prog Neurobiol. 2014;115:210-45. 
10. Wang B, Cao W, Biswal S, Doré S. Carbon monoxide-activated Nrf2 pathway leads to protection against permanent focal cerebral ischemia. Stroke. 2011;42(9):2605-10.

11. Jung E, Koh SH, Yoo M, Choi YK. Regenerative Potential of Carbon Monoxide in Adult Neural Circuits of the Central Nervous System. Int J Mol Sci. 2020;25(7):2273. 21(.

12. Choi YK, Maki T, Mandeville ET, Koh SH, Hayakawa K, Arai K, Kim YM, Whalen MJ, Xing C, Wang X, Kim KW, Lo EH. Dual effects of carbon monoxide on pericytes and neurogenesis in traumatic brain injury. Nat Med 2016 Sep 26. doi:10.1038/nm.4188. [Epub ahead of print].

13. Romao CC, Blattler,WA.,Seixas,JD, Bernardes,G.J.

14. Developing drug molecules. for therapy with carbon monoxide. ChemSocRev. 2012;41:3571-83.

15. Xie Z, Han P, Cui Z, Wang B, Zhong Z, Sun Y, Yang G, Sun Q, Bian L. Pretreatment of Mouse Neural Stem Cells with Carbon Monoxide-Releasing Molecule-2 Interferes with NF-кB p65 Signaling and Suppresses Iron Overload-Induced Apoptosis. Cell Mol Neurobiol. 2016;36(8):1343-51.

16. Lundqvist J, El Andaloussi-Lilja J, Svensson C, Gustafsson Dorfh H. Forsby A Optimisation of culture conditions for differentiation of C17.2 neural stem cells to be used for in vitro toxicity tests. Toxicol In Vitro. 2013;27:1565-9.

17. Lee CC, Yang HL, Way TD, Kumar KJ, Juan YC, Cho HJ, Lin KY, Hsu LS, Chen SC, Hseu YC. Inhibition of cell growth and induction of apoptosis by Antrodia camphorata in HER-2/neu-overexpressing breast cancer cells through the induction of ROS, depletion of HER-2/neu, and disruption of the PI3K/Akt signaling pathway, Evid. Based Complement Alternat Med. 2012;2012:702857. doi:10.1155/2012/702857. Epub 2012 Jun 3.

18. Tang Y, Wang J, Lin X, Wang L, Shao B, Jin K, Wang Y, Yang GY. Neural stem cell protects aged rat brain from ischemia-reperfusion injury through neurogenesis and angiogenesis. J Cereb Blood Flow Metab. 2014 Jul;34(7):1138-47.

19. Sakata H, Niizuma K, Yoshioka H, Kim GS, Jung JE, Katsu M, Narasimhan P, Maier CM, Nishiyama Y, Chan PH. Minocycline-Preconditioned Neural Stem Cells Enhance Neuroprotection after Ischemic Stroke in Rats. J Neurosci. 2012;32:3462-73.

20. Sucak GT, Yegin ZA, Ozkurt ZN, Aki SZ, Yağci M. Iron Overload: Predictor of Adverse Outcome in Hematopoietic Stem Cell Transplantation Transplantation Proceedings,2010;42, 1841-1848.

21. Balogh E, Tolnai E, Nagy B Jr, Nagy B, Balla G, Balla J, Jeney V. Iron overload inhibits osteogenic commitment and differentiation of mesenchymal stem cells via the induction of ferritinãubiochim. Biophys Acta. 2016;1862(9):1640-9.

22. Pahl HL. Activators and target genes of Rel/NF-kappaB transcription factors. Oncogene. 1999;18(49):6853-66.

23. Qing Y, Liang Y, Du Q, et al. Apoptosis induced by trimethyltin chloride in human neuroblastoma cells SY5Y is regulated by a balance and cross-talk between NF-kappaB and MAPKs signaling pathways. Arch Toxicol. 2013;87:1273-85.

24. Kalmar B, Greensmith L. Induction of heat shock proteins for protection against oxidative stress. Adv Drug Deliv Rev. 2009;61:310-8. 
25. Li SW, Liu CM, Guo J, Marcondes AM, Deeg J, Li X, Guan F. Iron overload induced by ferric ammonium citrate triggers reactive oxygen species-mediated apoptosis via both extrinsic and intrinsic pathways in human hepatic cells. Hum Exp Toxicol. 2016;35(6):598-607.

26. Rodríguez Diez G, Sánchez Campos S, Giusto NM. G.A. Salvador Specific roles for Group V secretory PLA2 in retinal iron-induced oxidative stress. Implications for age-related macular degenerationExperimental. Eye Research. 2013;113:172-81.

27. Jang JH, Surh YJ. Beta-amyloid-induced apoptosis is associated with cyclooxygenase-2 upregulation via the mitogenactivated protein kinase-NF-kappaB signaling pathway. Free Radic Biol Med. 2005;38(12):1604-13.

28. Zhu C, Liu Z, Gui L, Yao W, Qian W, Zhang C. Mutated IkappaBalpha represses proliferation of immortalized neural progenitor cells and prevents their apoptosis after oxygen-glucose deprivation. Brain Res. 2008;1244:24-31.

29. Kutuk O, Basaga H. Aspirin prevents apoptosis and NF-kappaB activation induced by $\mathrm{H} 2 \mathrm{O} 2$ in Hela cells. Free Radic Res. 2003;37(12):1267-76.

30. Kensler TW, Wakabayashi N, Biswal S. Cell survival responses to environmental stresses via the Keap1-Nrf2- ARE pathway. Annu Rev Pharmacol Toxicol. 2007;47:89-116.

31. Slocum S, Kensler T. Nrf2: control of sensitivity to carcinogens. Arch Toxicol. 2011;85:273-84.

32. Doré S. Neuroprotective effert of carbon monoxide and Nrf2 in cerebral ischemia. Springerplus. 2015;4(Suppl 1):L44.

33. Chi PL, Lin CC, Chen YW, Hsiao LD, Yang CM. CO Induces Nrf2-Dependent Heme Oxygenase-1 Transcription by Cooperating with Sp1 and c-Jun in Rat Brain Astrocytes. Mol Neurobiol. 2015;52(1):277-92.

34. Sakata H, Niizuma K, Yoshioka H, Kim GS, Jung JE, Katsu M, Narasimhan P, Maier CM, Nishiyama Y, Chan PH. Minocycline-Preconditioned Neural Stem Cells Enhance Neuroprotection after Ischemic Stroke in Rats. J Neurosci. 2012;32:3462-73.

35. Johansson K, Cebula M, Rengby O, Dreij K, Carlström KE, Sigmundsson K, Piehl F, Arnér ES. Cross Talk in HEK293 Cells Between Nrf2, HIF,and NF-jB Activities upon Challenges with RedoxTherapeutics Characterized with Single-Cell Resolution .Antioxid Redox Signal. 2015 Nov 11. [Epub ahead of print].

36. Li W, Khor TO, Xu C, Shen G, Jeong WS, Yu S, et al. Activation of Nrf2-antioxidant signaling attenuates NFkappaB-inflammatory response and elicits apoptosis. Biochem Pharmacol. 2008;76:1485-9.

37. Jin W, Zhu L, Guan Q, Chen G, Wang QF, Yin HX, Hang CH, Shi JX, Wang HD. Influence of Nrf2 genotype on pulmonary NF-kappaB activity and inflammatory response after traumatic brain injury. Ann Clin Lab Sci. 2008;38:221-7.

38. Joanna D, Wardyn AH, Ponsford. and Christopher M. Sanderson. Dissecting molecular cross-talk between Nrf2 and NF-kB response pathwaysBiochem. Soc Trans. 2015;43:621-6.

39. Stuart A, Rushworth. and David J. MacEwan.The Role of Nrf2 and Cytoprotection in Regulating Chemotherapy Resistance of Human Leukemia Cells. Cancers. 2011;3:1605-21. 
40. Paur I, Balstad TR, Kolberg M, Pedersen MK, Austenaa LM, Jacobs DR, Blomhoff R. Extract of oregano, coffee, thyme, clove, and walnuts inhibits NF-KB in monocytes and in transgenic reporter mice. Cancer Prev Res. 2010;3:653-63.

41. Mukherjee S, Ghosh S, Choudhury S, Adhikary A, Manna K, Dey S, Sa G, Das T, Chattopadhyay S. Pomegranate reverses methotrexate-induced oxidative stress and apoptosis in hepatocytes by modulating Nrf2-NF-KB pathways. J Nutr Biochem. 2013;24(12):2040-50.

42. Alba Minellia S, Grottelli A, Mierla F, Pinnen I, Cacciatore I, Bellezza. Cyclo(His-Pro) exerts antiinflammatory effects by modulating NF-KB and Nrf2 signaling. The International Journal of Biochemistry Cell Biology. 2012;44:525-35.

43. Yabluchanskiy A, Sawle P, Homer-Vanniasinkam S,et al. CORM-3, a carbon monoxide-releasing molecule, alters the inflammatory response and reduces brain damage in a rat model of hemorrhagic stroke. Crit Care Med. 2012;40:544-52.

\section{Figures}

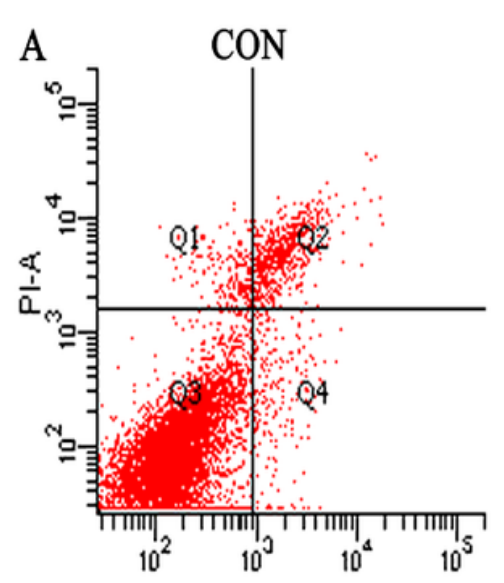

FITC-A

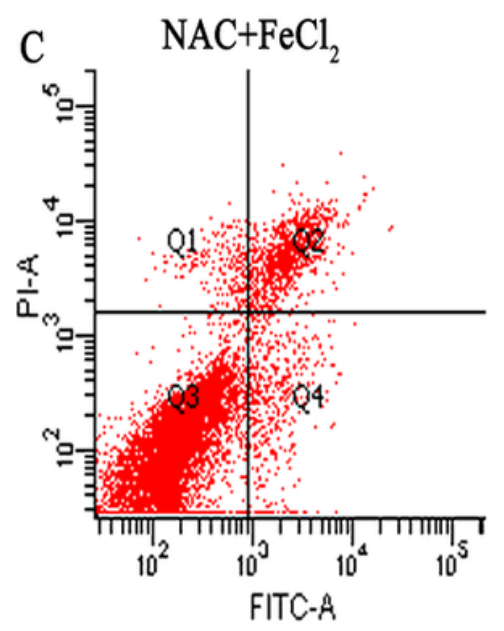

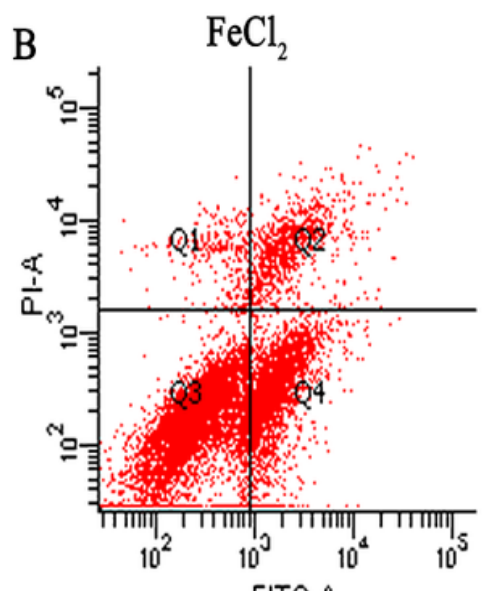

FITC-A

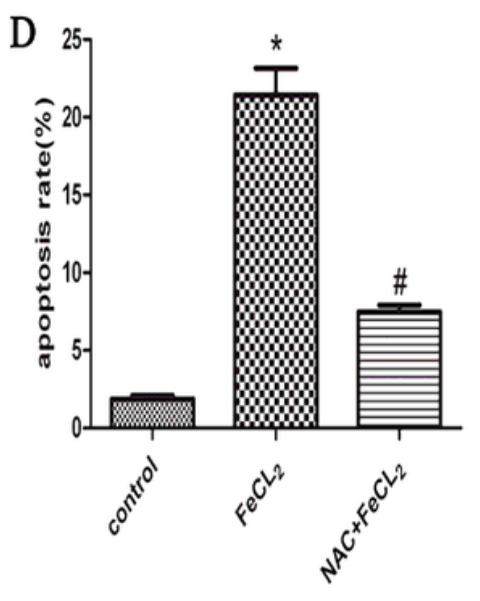

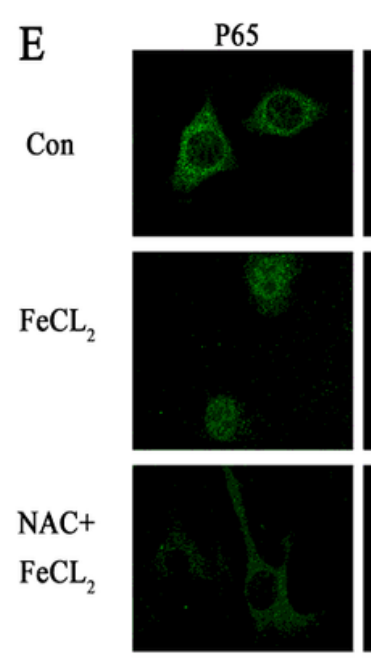

$\underset{\text { Cyto P65 }}{\text { F }}$ GAPDH

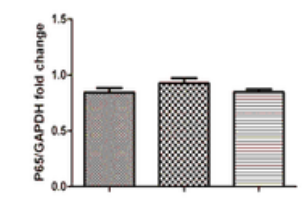

FeCL2

NAC
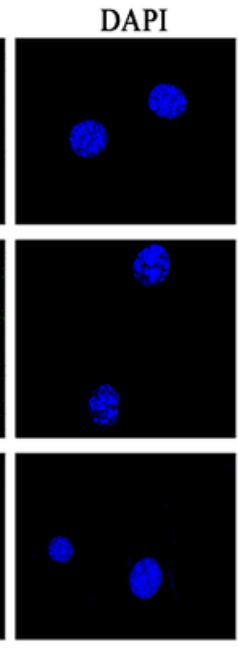

$\mathrm{G}_{\text {Nucleus }}$
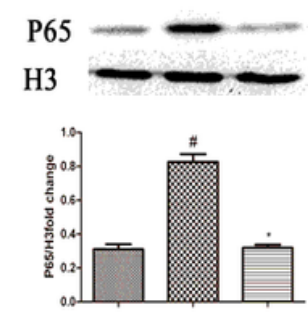

FeCL2 - ++

NAC - - $\quad+$

Figure 1 
Role of ROS in NSCs challenged by iron overload in vitro. (A) apoptosis of NSCs without any insult; (B) apoptosis of NSCs challenged by 24h iron overload without NAC coincubation. (C) Apoptosis of the NSCs

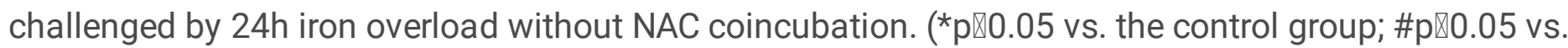
$\mathrm{FeCl} 2$ group). Data represent the mean $\pm S D(n=3)$. (E) Representative images showing co-incubation with $10 \mu \mathrm{M}$ NAC inhibited the translocation of NF-KB induced by iron overload. Double staining with DAPI (blue) and NF-KB (green). (F), (G) Representative bands and quantification of NF-KB in the cytoplasm and

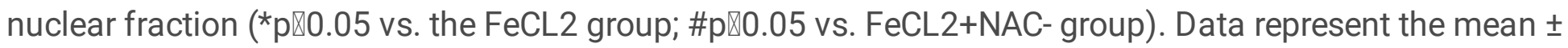
SD $(n=3)$. Bar25 $\mu \mathrm{m}$

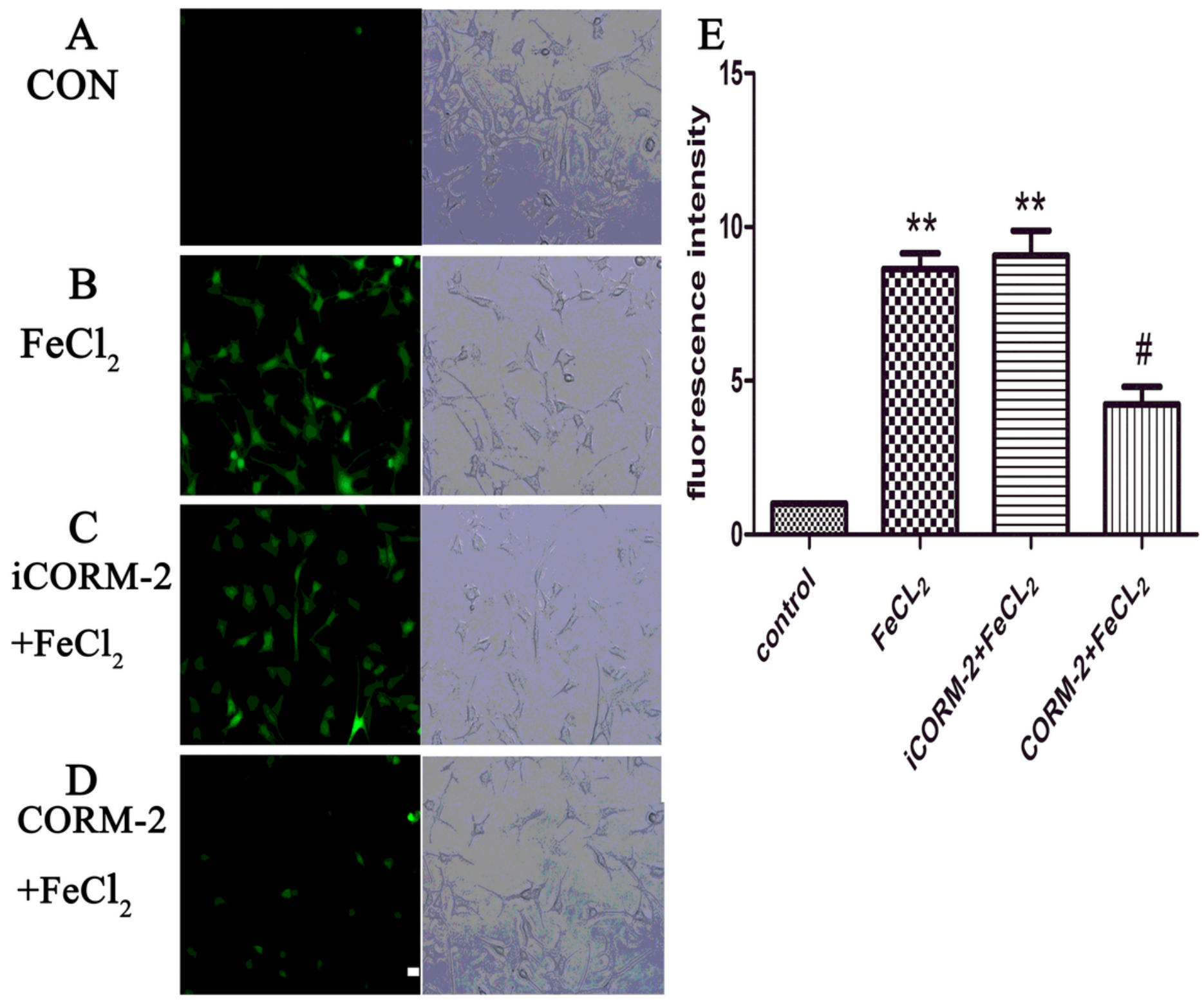

\section{Figure 2}

Effect of CO on ROS generation. Cells were challenged by $500 \mu \mathrm{M}$ FeCL2 with or without pretreatment with $50 \mu \mathrm{M}$ CORM-2/ICORM-2 for the indicated time periods and followed by incubation with $10 \mu \mathrm{M}$ ROS- 
sensitive fluorophore H2DCFDA.Quantitative induction is shown as an increase in the treated cells relative to the control. (** $\mathbb{\star} \varangle 0.001$ vs. the control group; \#p $₫ 0.05$ vs. the FeCL2 group). Data represent the mean \pm $\operatorname{SD}(\mathrm{n}=3)$. Bar $25 \mu \mathrm{m}$.

A

Con

$\mathrm{FeCL}_{2}$
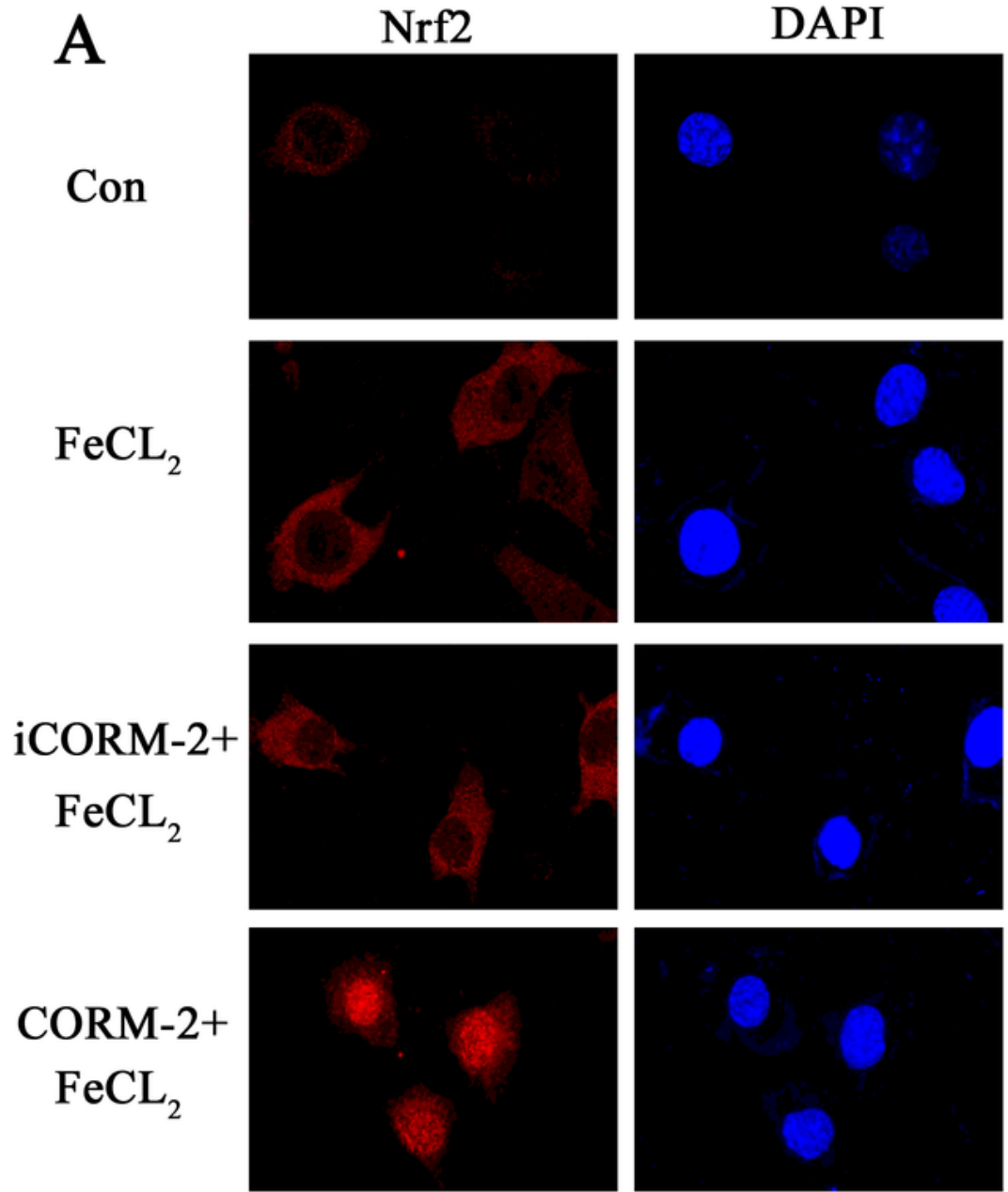

B Cyto

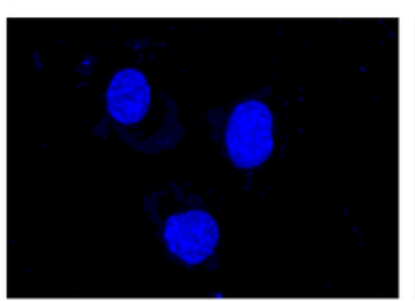

CORM-2+ $\mathrm{FeCL}_{2}$

$$
\text { Nrf2 }
$$

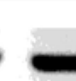

GAPDH

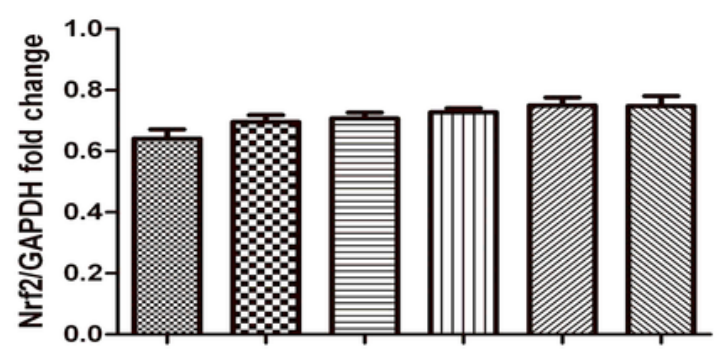

C Nrf2

\section{Nucleus}
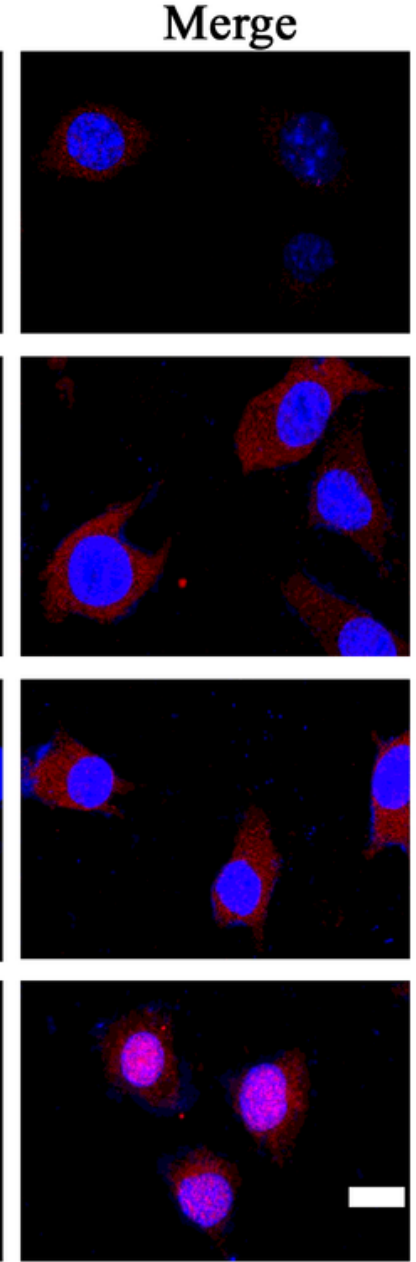

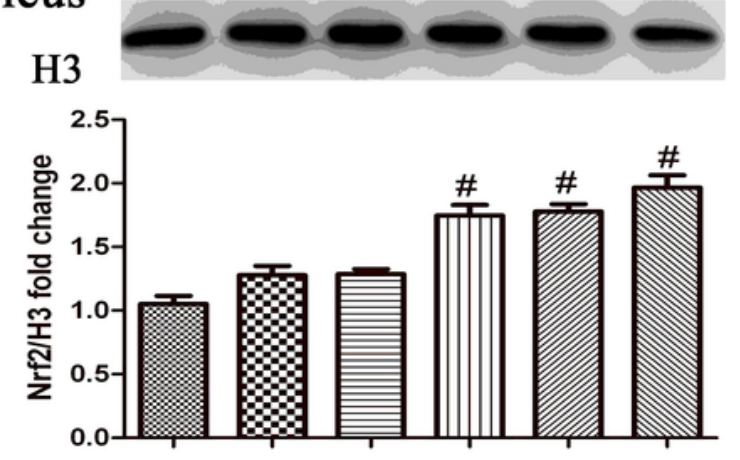

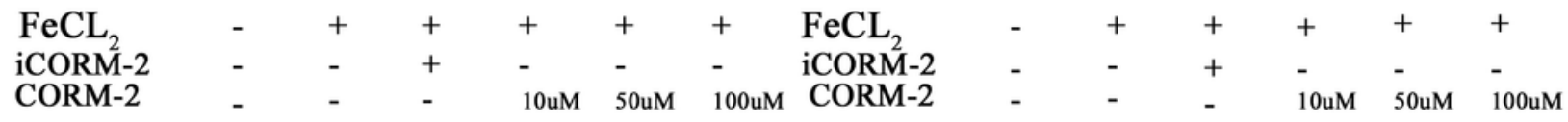

\section{Figure 3}

Effects of CO preconditioning on Nrf2 signaling pathway in NSC challenged by iron overload in vitro. (A) Representative images showing CO preconditioning (50 $\mathrm{MM}$ CORM-2) triggered activation of Nrf2. Double 
staining with DAPI (blue) and Nrf2 (red). (B), (C) Representative bands and quantification of Nrf2 in the cytoplasm and nuclear fraction (\#p $₫ 0.05$ vs. the $\mathrm{FeCl} 2$ group or iCORM-2 group). Data represent the mean \pm SD $(n=3)$. Bar $25 \mu \mathrm{m}$.

A
NQ0-1 Actin

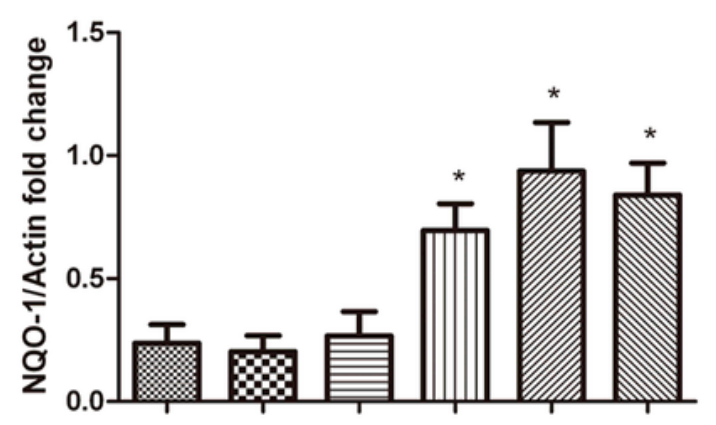

0h 2h 4h 6h 12h 24h

\section{B}

\section{NQO-1}

Tublin

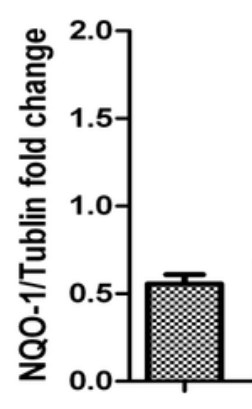

$\mathrm{FeCL}_{2}(500 \mu \mathrm{M})$
iCORM-2 $(\mu \mathrm{M})$
CORM-2( $\mu \mathrm{M})$
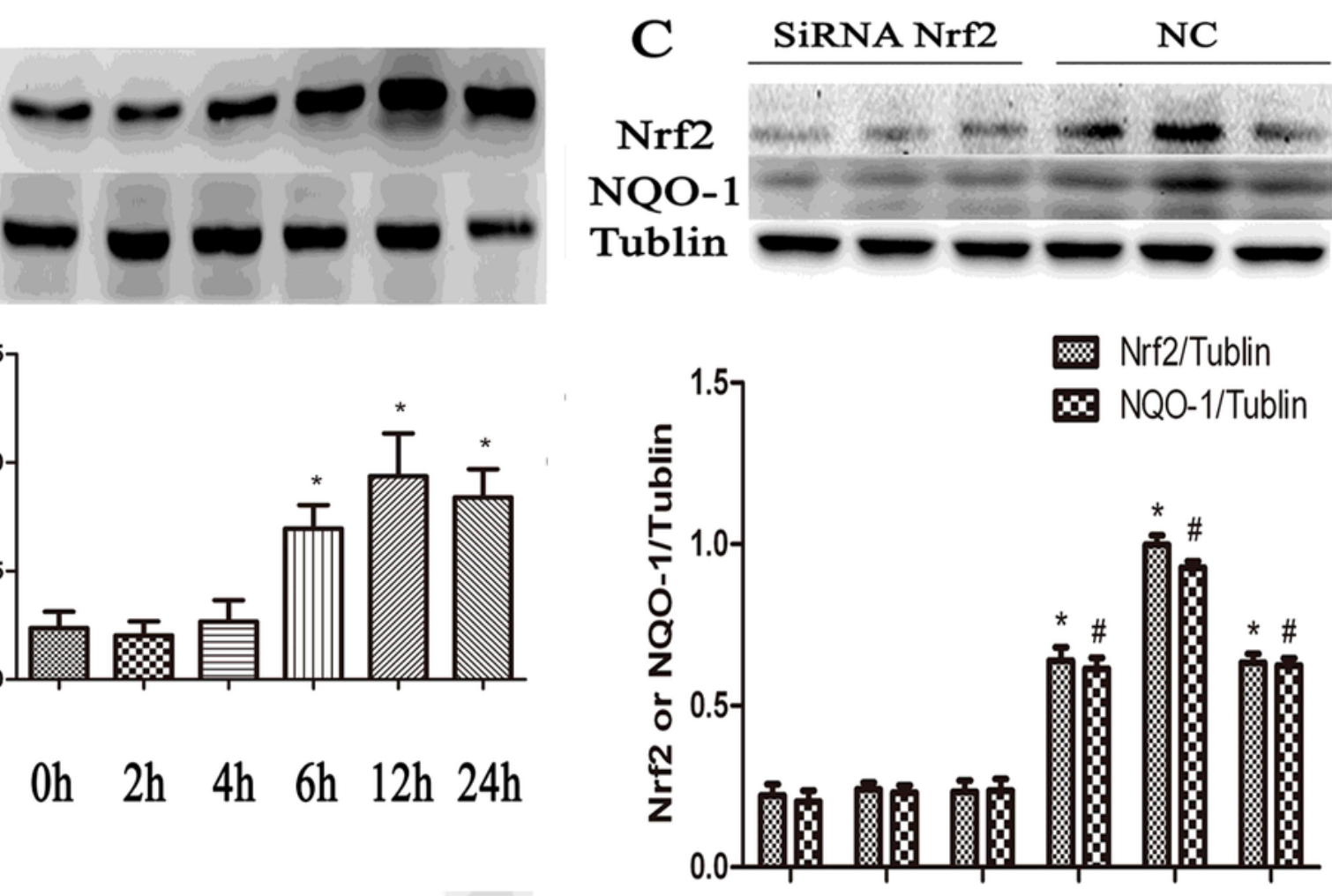

Figure 4

Effects of CO on antioxidant protein level in NSCs in vitro. (A) Cells were pretreated with $50 \mu \mathrm{M}$ CORM-2 for the indicated time periods. Representative bands and quantification revealed that $\mathrm{CO}$ preconditioning 
induced higher expression of the Nrf2-regulated antioxidant genes NQO1 in a time dependant manner. ( ${ }^{\star} \mathrm{p}$ $\$ 0.05$ vs. the control(Oh) group). $(n=3)(B) C O$ preconditioning upregulated the expression of NQ01in

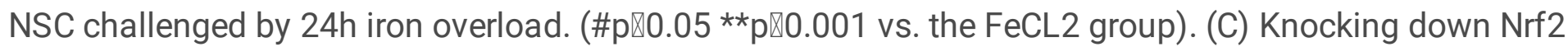
by Nrf2 SiRNA reverses the upregulation of NQ01. (D) Representative bands in the nuclear fraction showing $\mathrm{CO}$ suppresses NFKB activation through upregulation of Nrf2 ( ${ }^{\star} \mathrm{p} \otimes 0.05 ; \# \mathrm{p} \otimes 0.05$ vs. NC group). Data represent the mean $\pm S D(n=3)$.
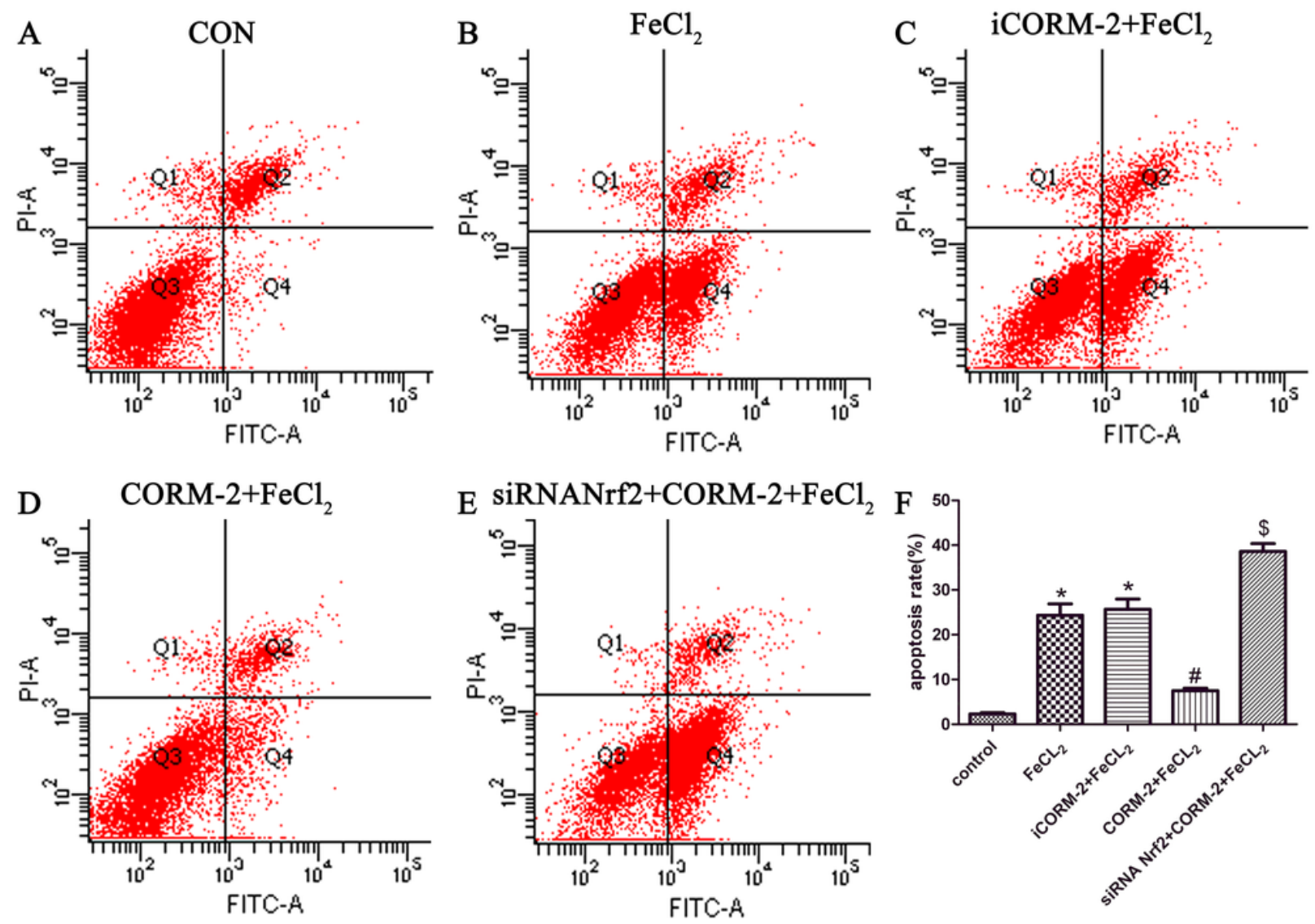

\section{Figure 5}

Inhibition of Nrf2 abolished CO-induced cytoprotection in vitro. Apoptosis of the NSCs was analyzed by flow cytometry after $24 \mathrm{~h}$ iron overload. Iron overload induced significant apoptosis in NSCs(A,B,F); CO preconditioning significantly attenuated the iron overload induced apoptosis(C,D,F); A significant increase of apoptosis in NSC knocked down Nrf2 with SiRNA after $24 \mathrm{~h}$ of iron overload(E,F). ( ${ }^{\star} \mathrm{p} \otimes 0.05 \mathrm{vs}$. the FeCl2 group or iCORM-2 group; \#p $₫ 0.05$ vs. the control group; $\$ p \rrbracket 0.01$ vs CORM-2 group.). Data represent the mean $\pm S D(n=3)$. 

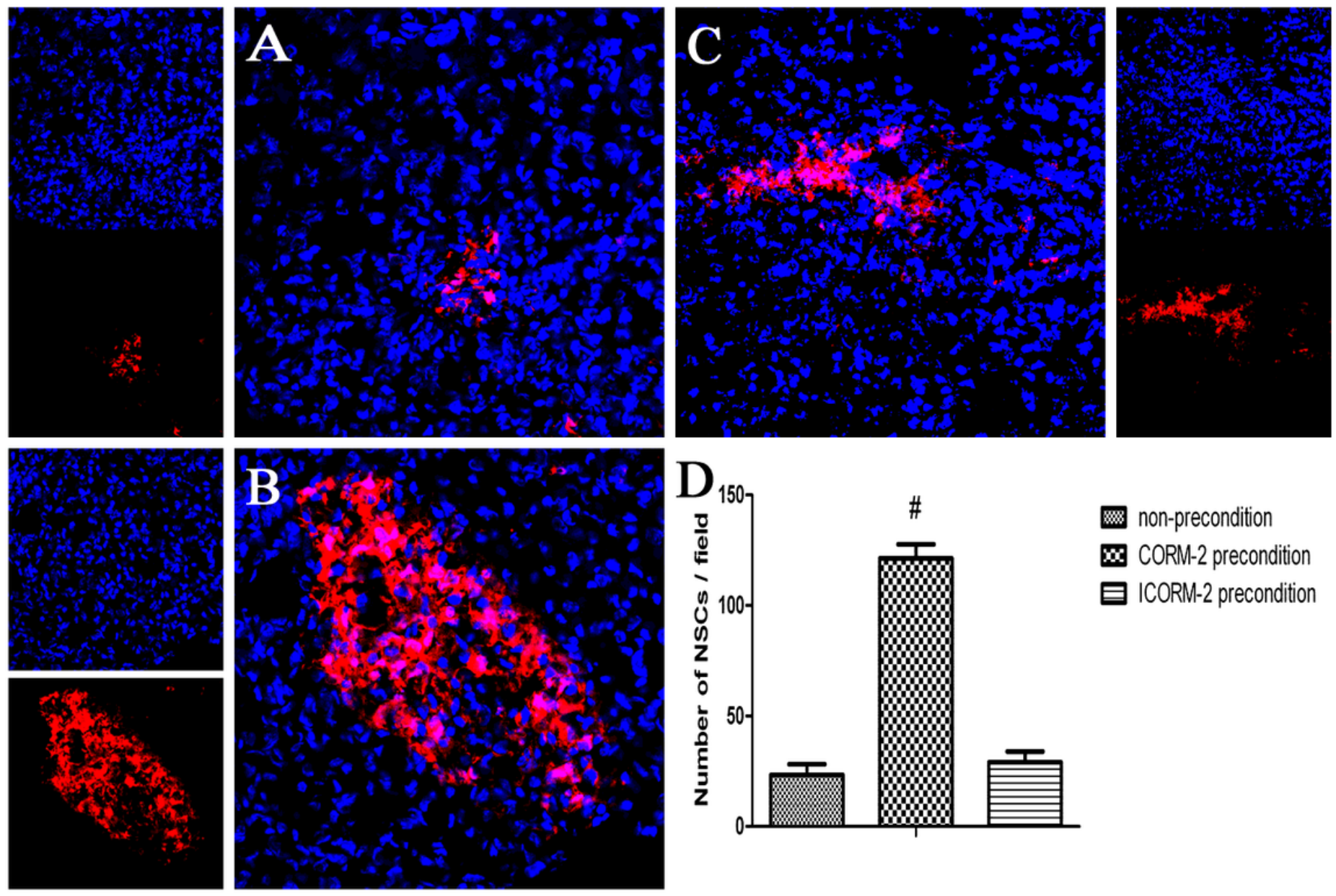

\section{Figure 6}

Increased cell survival in vivo. Fluorescent staining with RFP (red) revealed that the grafted cells surrounded the HS lesion 30 days after transplantation (A) NSCs in non-preconditioned group; (B) more NSCs in CO preconditioned group; (C) NSCs in iCORM-2-preconditioned group. (\#p囚0.05 vs. nonpreconditioned group or iCORM-2-preconditioned group) 